\title{
AUTOFLUORESCENCE SPECTROSCOPY OF A HUMAN GASTROINTESTINAL CARCINOMA CELL LINE Design of Optical Sensors for the Detection of Early Stage Cancer
}

\author{
D. S. Ferreira ${ }^{1}$, M. Henriques ${ }^{2}$, R. Oliveira ${ }^{2}$, J. H. Correia ${ }^{1}$ and G. Minas ${ }^{1}$ \\ ${ }^{1}$ Algoritmi Centre, Universidade do Minho, Campus Azurém, 4800-058 Guimarães, Portugal \\ ${ }^{2}$ Department of Biological Engineering, Universidade do Minho, Campus Gualtar, 4710-057 Braga, Portugal \\ debora@dei.uminho.pt,mcrh@deb.uminho.pt,gminas@dei.uminho.pt,roliveira@deb.uminho.pt \\ higino.correia@dei.uminho.pt
}

Keywords: Autofluorescence, Fluorophores, Cancer, Optical sensors.

Abstract: Human tissues show autofluorescence (AF) emission spectra when excited by ultraviolet or shortwavelength visible light. The intensity and shape of these spectra are dependent on the tissues pathological state and, therefore, its measurement gives information about the degree of malignant transformations that could lead to cancer. In this article, it is characterized the AF spectra of one human gastrointestinal carcinoma cell line (CACO-2). The obtained results showed significant AF signal for the presence of amino acids. The spectral information obtained can be used for the design of fluorescence optical sensors that will be incorporated on an endoscopic capsule, for measuring the AF emission spectra of normal and cancer cells. This integrated optical system will innovate on the diagnosis of early stage cancer.

\section{INTRODUCTION}

Cancer of the gastrointestinal (GI) tract is the second most common cause of cancer death in the United States and industrialized countries. Patients with a family history of colon cancer, familial polyposis, long-standing inflammatory bowel disease, and Barrett's esophagus are at high risk for developing this kind of cancer. The new innovations in endoscopy, including capsule endoscopy, made the GI tract one of the most frequently and completely examined system. Despite the greater access, early detection of neoplasia is difficult during routine endoscopy due to the absence of typical morphological structures and, as a result, is still limited to blind random biopsies in patients at high risk of developing cancer, in a both inefficient and costly process. Therefore, more sensitive endoscopic screening tools, which enable differentiation between premalignant and benign lesions during endoscopy, are of scientific and clinical interest (Prosst, 2002; Banerjee, 2004; Georgakoudi, 2006).

When human tissues are illuminated with ultraviolet or short wavelength visible light, they emit fluorescence light of a longer wavelength (Figure 1). This tissue 'autofluorescence' (AF) arises from endogenous molecules within the tissue, called fluorophores (Haringsma, 1999; DaCosta, 2002).

The GI tract tissues are composed by a complex combination of several fluorophores that occur in different concentrations and at different depths. The mucosa, submucosa and muscularis propria have distinct fluorophore compositions, so that even though each fluorophore has a distinct fluorescence spectrum, the total fluorescence measured comprises contributions from the fluorophores in the various layers. Hence, excitation and fluorescence emission wavelength bands are often broad, relatively featureless and overlap with one another, so that identifying individual fluorophores in a given tissue spectrum is difficult (Haringsma, 1999; DaCosta, 2002).

Some research groups have explicitly studied the AF emission of established cell lines (DaCosta, 2005). These studies, by the use of single living cells, allow the isolation of the contribution of intracellular fluorescence in the tissular emission, through the elimination of the extracellular matrix emission and the influence of light absorption and scattering. Therefore, such studies provide a better understanding of the biochemical changes associated with malignant transformation. Also, cellular 
reference spectra are needed for the modeling of the tissue fluorescence, in view of spectral quantification which should be very useful for diagnostic purposes. To quantify the relatively weak AF created by one single fluorophore, sophisticated algorithms have been developed (Stepp, 1998; Prosst, 2002; Villette, 2006).

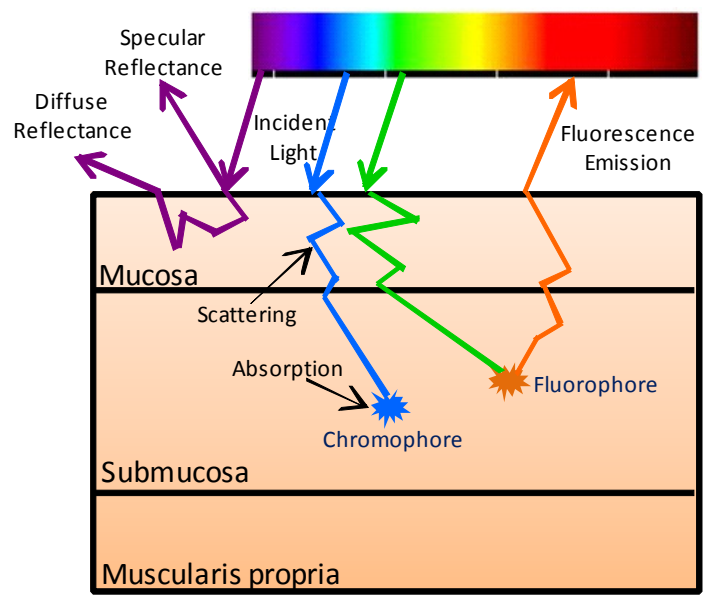

Figure 1: Light-tissue interactions include reflectance, scattering, absorption and fluorescence. Tissue fluorescence is originated by the absorption of light by fluorophores.

Among the significant fluorophores in the GI tract are: tryptophan and tyrosine (aromatic amino acids present in cells); NADH and NADPH (cellular metabolism related coenzymes), which assist oxidation and reduction processes and are found mainly in mitochondria; and flavins and flavin nucleotides, which are mostly bound to enzymes and concentrated in the mitochondria (DaCosta, 2005). Each of these fluorophores has its characteristic excitation and emission spectrum (Table 1). Thus, different excitation wavelengths result in the activation of different fluorophores.

Table 1: Excitation and emission maxima of some endogenous fluorophores (DaCosta, 2002).

\begin{tabular}{|l|c|c|}
\hline \multicolumn{1}{|c|}{$\begin{array}{c}\text { Endogenous } \\
\text { fluorophores }\end{array}$} & $\begin{array}{c}\text { Excitation } \\
\text { maxima }(\mathrm{nm})\end{array}$ & $\begin{array}{c}\text { Emission } \\
\text { maxima }(\mathrm{nm})\end{array}$ \\
\hline $\begin{array}{l}\text { Amino acids } \\
\text { Tryptophan }\end{array}$ & 280 & 350 \\
Tyrosine & 275 & 300 \\
Phenylalanine & 260 & 280 \\
\hline Metabolic cofactors & & \\
FAD, Flavins & 450 & 515 \\
NADH & 350 & 450 \\
NADPH & 336 & 464 \\
\hline
\end{tabular}

Over the last decade a number of optical techniques have been developed to try to detect early GI neoplastic lesions. These optically-based methods have the potential to detect the very earliest mucosal changes at the micro structural and molecular levels (Haringsma, 1999). Light induced autofluorescence spectroscopy, for example, is based on the analysis of the fluorescence emission of endogenous fluorophores, providing information that can be used to characterize changes that take place as tissues become diseased. It involves delivery of the excitation light via placement of a fiber-optic probe in direct contact with tissue. The fluorescence is collected by the same probe and delivered to the input of a spectrometer. Achieving discrimination between diseased and healthy tissue relies upon the identification of differences either in emission intensity, spectral distribution, fluorescence lifetime, or crucially, a combination of these (Stringer, 2004; Georgakoudi, 2006).

The capsule endoscopy is a relatively recent method for GI tract evaluation that uses an endoscopic capsule (EC). Since, presently, some of the most promising methods for detecting cancer at an early stage are based on tissue's fluorescence, the EC can play an important role in this kind of diagnosis, once it can be equipped with miniaturized fluorescence CMOS optical sensors. These sensors should be incorporated in the EC for measuring the AF emission spectra of normal and cancer cells, and should have the higher quantum efficiency in a defined spectral range (Delvaux, 2006; Dias, 2007). This integrated system will certainly innovate in the diagnosis of GI cancer. It is important to notice that current endoscopic capsules do not have added diagnostic functions, such as spectroscopic analysis, which are commonly available in conventional endoscopes. Therefore these developments will be a major step towards creating a new platform for diagnosis.

In this article, it is characterized the $\mathrm{AF}$ spectra of one human GI carcinoma cell line (CACO-2). The results obtained showed significant AF signal for the presence of amino acids, namely tryptophan. The resulting spectral information of the CACO-2 cell line is a good initial approach for the design of fluorescence optical sensors, to be incorporated on an EC. This study was performed using spectrofluorimetry on cell monolayers, with several excitation wavelengths, resulting in several fluorophores emission spectra. Some of the reported results appeared promising, being in accordance with previously published results. 


\section{EXPERIMENTAL}

\subsection{Human Carcinoma Cell Line Culture}

The experimental studies were performed using one human GI carcinoma cell line - CACO-2 - that was purchased from the European Collection of Cell Cultures (ECACC). These cells were grown in $88 \%$ Minimum Essential Medium Eagle's (EMEM), supplemented with 10\% Fetal Bovine Serum (FBS), $1 \%$ Non Essential Amino Acids (NEAA), and 1\% Glutamine $(2 \mathrm{mM})$ in a humidified atmosphere, at $37^{\circ} \mathrm{C}$ and $5 \% \mathrm{CO}_{2}$. Cells were grown in $25 \mathrm{~cm}^{2}$ flasks to $70-80 \%$ confluence and their morphology was routinely inspected.

\subsection{Preparation of CACO-2 Cell Line for Spectrofluorimetry}

The flasks of cultured cells were examined under the inverted microscope to observe cell confluency. After that, the old medium was removed and discarded and cells were washed with warm $\left(37^{\circ} \mathrm{C}\right)$ phosphate-buffered solution (PBS). A small volume of trypsin $(\sim 1 \mathrm{~mL})$ solution was added to the flasks and the flasks were placed back in an incubator at $37^{\circ} \mathrm{C}$. Five to ten minutes after, cells were examined under the inverted microscope to confirm that most of the cells had rounded up and were detaching from the flask surface. Finally, it was added $4 \mathrm{~mL}$ of fresh growth medium to the cell suspensions. The number of viable and dead cells $/ \mathrm{mL}$ was determined using a Neubauer haemocytometer and the trypan blue dye exclusion method.

A total of eight experiments were performed for the CACO-2 cell line. To support the cells for these experiments it was used glass cover slips placed inside 6-well plates; after that the final cell suspensions were divided and placed within these 6well plates. The cells used were grown as monolayers over the cover slips. After 24,48 or 72 hours (depending on the experiment), cover slips were collected from the 6-well plates and packed in Petri dishes, with $3 \mathrm{~mL}$ of PBS solution, for the experiments. Spectral analysis was then performed within less than half an hour after cells were placed in Petri dishes.

\subsection{Spectrofluorimetry of CACO-2 Cell Line}

Fluorescence spectra were performed on human carcinoma cell monolayers. The samples were measured using a SPEX ${ }^{\circledR}$ FluoroLog ${ }^{\circledR}$ - a high sensitivity spectrofluorometer with a SPEX1680 $(0,22 \mathrm{~m})$ Double Spectrometer. The excitation light was provided by a Xenon lamp of $450 \mathrm{~W}$ with a DC 450RAM power supply, from EUROSEP Instruments. The equipment was connected to a computer to control and collect the data. The slit widths of both spectrometers were adjusted in order to provide a good spectral resolution in the excitation and emission path.

\subsection{Fluorescence Spectra of CACO-2 Cell Line}

Emission spectra were recorded from 270-690 nm, for an excitation wavelength ranging from 260 to $350 \mathrm{~nm}$. These excitation wavelengths were chosen in order to allow a comparison with data obtained from literature. Reference spectra were obtained with pure PBS solutions. All the spectra were normalized subtracting the reference spectra from the fluorescence spectra. The fluorescence emission spectra were also corrected for the spectral response of the spectrofluorometer. The recordings were performed for several cell concentrations, depending on the experiment $(24,48$ or 72 hours). These cells were maintained in PBS during the measurement, which could in some way put in risk cell viability. All measurements were performed under the same experimental conditions.

\section{RESULTS AND DISCUSSION}

The analysis of the two epithelial cell lines AF was performed using spectrofluorimetry on cell monolayers. The measurements were performed on cell monolayers to use epithelial cells as close as possible to their in vivo tissular physiological conditions, as cells grown as monolayers undergo less stress than cells in suspension. This was confirmed on previous studies on normal cells from primary cultures, which easily grow as adherent monolayers looking like an epithelium structure while showing a poor viability in suspension. Furthermore, cell monolayers can be considered as an optically thin medium, while the acquired spectra from cell suspensions are strongly affected by 
scattering, inducing spectral distortion (Villette, 2006).

The experiments started measuring the AF signal of the CACO-2 cell line, with 24,48 and 72 hours of growth, placed inside a 6-well plate and slightly immersed in PBS. The fluorescence measurements were made for different excitation wavelengths $\left(\lambda_{\mathrm{ex}}\right)$ - 260, 270, 280, 335, and $350 \mathrm{~nm}$ - and the emission was collected in the range $270-690 \mathrm{~nm}$. The average number of viable and dead cells was determined in each experiment (Table 2).

Table 2: Number of viable and dead CACO-2 cells, after 24,48 and 72 hours in culture.

\begin{tabular}{|c|c|c|c|}
\cline { 2 - 4 } \multicolumn{1}{c|}{} & $\begin{array}{c}\text { Time in culture } \\
\text { (hours) }\end{array}$ & Live & Dead \\
\hline \multirow{2}{*}{$\begin{array}{c}\text { Number of } \\
\text { cells } / \mathrm{mL}\end{array}$} & 24 & $16,5 \times 10^{4}$ & $1,25 \times 10^{4}$ \\
\cline { 2 - 4 } & 48 & $22,0 \times 10^{4}$ & $1,40 \times 10^{4}$ \\
\cline { 2 - 4 } & 72 & $63,5 \times 10^{4}$ & $2,50 \times 10^{4}$ \\
\hline
\end{tabular}

In all experiments, for $\lambda_{\text {ex }}$ of 260,275 and $280 \mathrm{~nm}$ the emission spectra are very similar in shape (Figures 2, 3 and 4) - all of them exhibit a broad emission band from 310 to $370 \mathrm{~nm}$, with a peak around $340 \mathrm{~nm}$, which is primarily caused by amino acids, namely tryptophan.

However, the AF intensity diverges among all the acquired spectra, being related with culture time. The maximum $\mathrm{AF}$ intensity is achieved for a 48 hours cell culture, decreasing then as time elapses. This can be explained by the number of viable and dead cells. From 24 to 48 hours there is a considerable increase in the number of viable cells, which is translated by AF signal intensification. From 48 to 72 hours, despite the increase in the number of viable cells, there is a reduction in $\mathrm{AF}$ signal intensity. This intensity drop (for the minimum value) can be justified not only by the raise in the number of dead cells, but also by an increase in cell fragility, as cells are in culture for a longer time, supplied by the same growth medium (it's important to notice that the culture medium was not discarded and replaced by fresh growth medium in the period of 72 hours).

For other spectra acquired using $\lambda_{\text {ex }}$ of 350 and $335 \mathrm{~nm}$, for the detection of NADH and NADPH, respectively, the resulting fluorescence intensity was approximately zero, when compared to the amino acids AF intensity (Figures 5 and 6). This may be justified by the fact that the AF emission from these fluorophores, although existing, is very weak and doesn't superimpose over the PBS signal (reference signal). Probably these molecules are not present in significant amounts to contribute to a high quality and strong signal. It may also be possible that some other molecules partly affect the measurements by absorbing the fluorescence or by emitting fluorescence in the same bandwidth. These results were very similar for all the experiments performed (24, 48 and 72 hours growth).

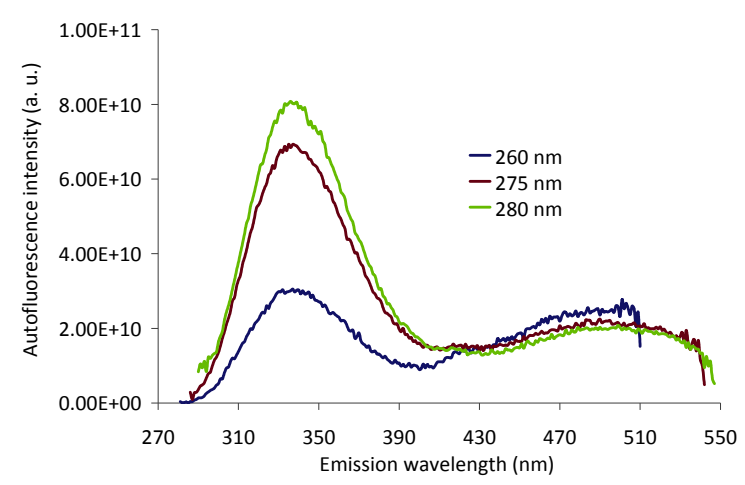

Figure 2: Autofluorescence emission spectra of a CACO-2 cell line, with 24 hours growth, obtained for different excitation wavelengths: 260,275 and $280 \mathrm{~nm}$.

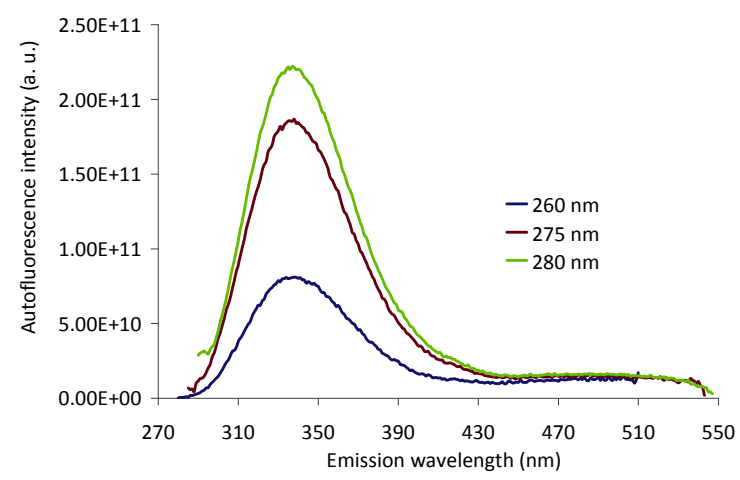

Figure 3: Autofluorescence emission spectra of a CACO-2 cell line, with 48 hours growth, obtained for different excitation wavelengths: 260,275 and $280 \mathrm{~nm}$.

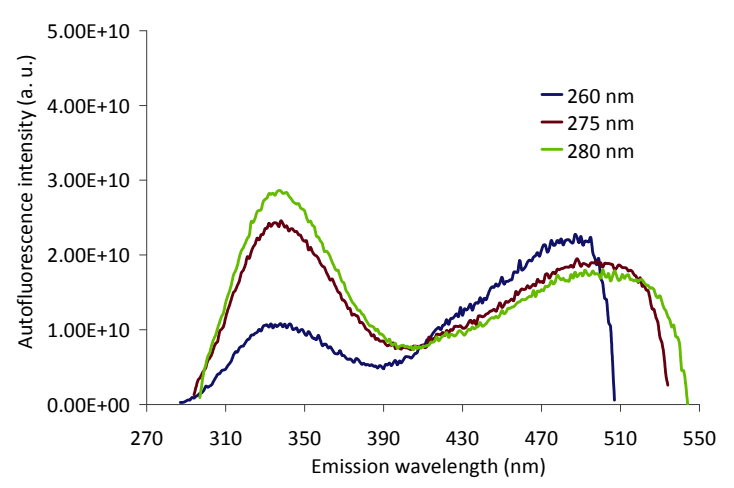

Figure 4: Autofluorescence emission spectra of a CACO-2 cell line, with 72 hours growth, obtained for different excitation wavelengths: 260,275 and $280 \mathrm{~nm}$. 
Recent studies have investigated the spectra of human intestinal mucosa using a spectrofluorometer. The measurements were performed using short wavelengths for excitation, and revealed utility in detecting neoplasia. The AF intensity increased with neoplasia and had the spectral line shape of tryptophan, indicating that $\mathrm{AF}$ emission from tryptophan might represent a viable approach to the detection of malignancy within colonic tissue (Banerjee, 2000, 2002). A different study had confirmed that the amount of amino acid related fluorescence (emission between 300 and $380 \mathrm{~nm}$ ) is greater in adenomatous polyps than in the normal tissues (DaCosta, 2003). Therefore, it can be said that the results obtained, namely for amino acids $\mathrm{AF}$ emission, are a good initial approach for the design of optical sensors for the detection of GI early stage cancer.

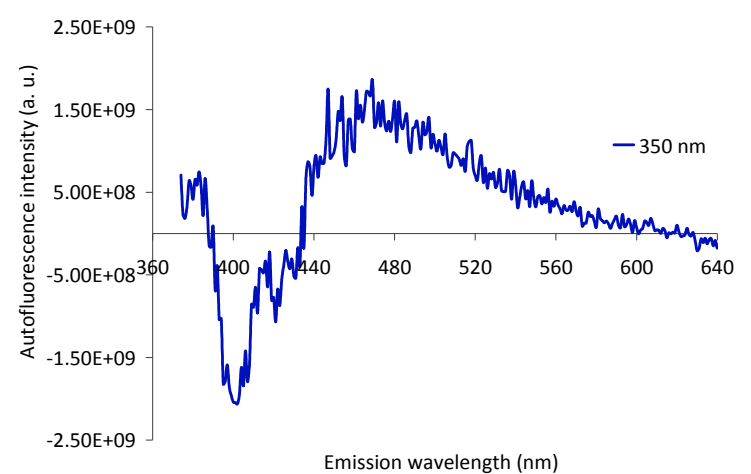

Figure 5: Autofluorescence emission spectra of a CACO-2 cell line, with 48 hours growth, obtained for an excitation wavelength of $350 \mathrm{~nm}$.

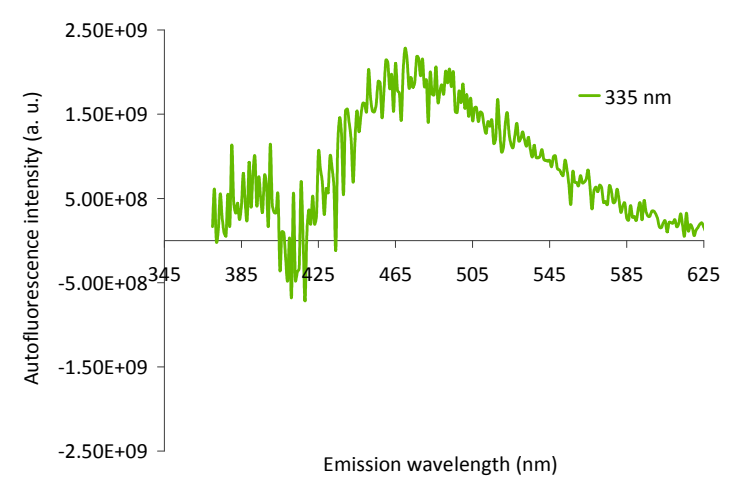

Figure 6: Autofluorescence emission spectra of a CACO-2 cell line, with 48 hours growth, obtained for an excitation wavelength of $335 \mathrm{~nm}$.

\section{CONCLUSIONS}

There is a great interest in developing autofluorescence-based spectroscopic systems for the detection of early stage cancers. Understanding the autofluorescent tissue components and how these components change in concentration and distribution with disease is essential in terms of optimizing diagnostic techniques. Several endogenous fluorophores have been identified and alterations in concentrations of these are suggested for discrimination of normal and malignant tissues.

In this article, it is characterized the AF spectra of one human GI carcinoma cell line (CACO-2), using several different excitation wavelengths, which targeted different molecular species. The obtained results showed significant AF signal for the presence of amino acids, namely tryptophan. The resulting spectral information of the CACO-2 cell line is consistent with previously published results of malignant colonic tissues, and is a good initial approach for the design of fluorescence CMOS optical sensors, to be incorporated on an EC, for the differentiation of normal and malignant tissues. It is important to notice, however, that when comparing fluorescence spectra of cell monolayers with those from in vivo or ex vivo tissues is has to be considered the possible changes due to the physicochemical microenvironment.

\section{REFERENCES}

Banerjee, B., Agarwal, S., Miedema, B., Perez R., Chandrasekhar, H. (2000). Use of a shorter wavelength autofluorescent band to separate adenomatous from hyperplastic polyps of the colon. Gastrointestinal Endoscopy, 51, AB149.

Banerjee, B., Chandrasekhar, H. (2002). Use of a single autofluorescence emission ratio for the detection of colonic neoplasia. Gastrointestinal Endoscopy, 55, AB129.

Banerjee, B., Henderson, J., Chaney, T., Davidson, N. (2004). Detection of Murine Intestinal Adenomas Using Targeted Molecular Autofluorescence. Digestive Diseases and Sciences, 49, 54-59.

DaCosta, R. S., Wilson, B. C., Marcon, N. E. (2002). New optical technologies for earlier endoscopic diagnosis of premalignant gastrointestinal lesions. Journal of Gastroenterology and Hepatology, 17, S85-S104.

DaCosta, R. S., Andersson, H., Wilson, B. C. (2003). Molecular Fluorescence Excitation-Emission Matrices Relevant to Tissue Spectroscopy. Photochemistry and Photobiology, 78, 384-392.

DaCosta, R. S., Andersson, H., Cirocco, M., Marcon, N. E., Wilson, B. C. (2005). Autofluorescence 
characterization of isolated whole crypts and primary cultured human epithelial cells from normal, hyperplastic, and adenomatous colonic mucosa. Journal of Clinical Pathology, 58, 766-774.

Delvaux, M., Gay, G. (2006). Capsule endoscopy in 2005: facts and perspectives. Best Practice \& Research Clinical Gastroenterology, 20, 23-39.

Dias, R. A., Correia, J. H., Minas, G. (2007). CMOS Optical Sensors for being incorporated in Endoscopic Capsule for Cancer Cells Detection. Proceedings of ISIE 2007, 2747-2751.

Georgakoudi, I. (2006). The color of cancer. Journal of Luminescence, 119, 75-83.

Haringsma, J., Tytgat, G. (1999). Fluorescence and Autofluorescence. Baillière's Clinical Gastroenterology, 13, 1-10.

Prosst, R. L., Gahlen, J. (2002). Fluorescence diagnosis of colorectal neoplasms: a review of clinical applications. International Journal of Colorectal Disease, 17, 1-10.

Stepp, H., Sroka, R., Baumgartner, R. (1998). Fluorescence endoscopy of gastrointestinal diseases: basic principles, techniques, and clinical experience. Endoscopy, 30, 379-386.

Stringer, M., Moghissi, K. (2004). Photodiagnosis and fluorescence imaging in clinical practice. Photodiagnosis and Photodynamic Therapy, 1, 9-12.

Villette, S., Pigaglio-Deshayes, S., Vever-Bizet, C., Validire, P., Bourg-Heckly, G. (2006). Ultravioletinduced autofluorescence characterization of normal and tumoral esophageal epithelium cells with quantitation of NAD $(\mathrm{P}) \mathrm{H}$. Photochemical and Photobiological Sciences, 5, 483-492. 